HELMINTHOLOGIA, 53, 3: 224 - 232, 2016

\title{
Effect of muramyl dipeptide and alum adjuvants on immunization with Filarial multi antigen peptide vaccine in mice model
}

\author{
I. CHRISTIANA ${ }^{1}$, R. APARNAA ${ }^{1}$, R. ROHIT ${ }^{1}$, D. NAGESWARA RAO ${ }^{2}$,P. KALIRAJ ${ }^{1 *}$
}

${ }^{1}$ Centre for Biotechnology, Anna University, Chennai-600 025, TamilNadu, India, *E-mail: kaliraj55@yahoo.co.in; ${ }^{2}$ Department of Biochemistry, All India Institute of Medical Sciences, New Delhi 110029, India

\section{Article info}

Received Novcember 9, 2014 Accepted June 1, 2015

\begin{abstract}
Summary
Filarial thioredoxin and transglutaminase are enzymes that are secreted throughout the lifecycle of the parasites which are mandatory for the survival of the parasite. They are reported to be promising vaccine candidates, yet the limitation factors of these proteins to be developed as vaccines is their homology they share with the host proteins. Hence immunodominant epitopes from these proteins were constructed as peptides and immunised in mice model with Muramyl dipeptide (MDP) as adjuvant. Immunodominant epitopic portions from Filarial thioredoxin and transglutaminase which are non-homologous with host proteins were constructed as Multi Antigen Peptide (MAP) and assembled in an inert lysine core. The synthesised MAP was immunised with MDP as adjuvant in Balb/c mice model, humoral and cellular immune response were studied. Antibody titre levels for TT MAP with MDP was in par with alum as adjuvant in mice models. T cell responses of TT MAP with MDP showed a balanced $T_{H} 1 / T_{H} 2$ response. The $T_{H} 1$ cytokines namely IL-2 and IFN- $\gamma$ were also higher in TT MAP immunised groups with MDP as adjuvant whereas alum immunised groups was $\mathrm{T}_{H} 2$ biased. TT MAP admixed with MDP as adjuvant proves to be safe in mice model. Further vaccination studies are underway in permissive animal models to determine the role of TT MAP with MDP as adjuvant in protective immunity against $W$. bancrofti and $B$. malayi infections.
\end{abstract}

Keywords: Filariasis; Thioredoxin; Transglutaminase; Multi Antigen Peptide (MAP); Muramyl dipeptide (MDP); Adjuvant

\section{Introduction}

Lymphatic filariasis (LF) is a mosquito-borne parasitic infection responsible for long-term chronic morbidity in the form of lymphoedema, genital pathology (especially hydroceles), recurrent disabling fevers (lymphangitis) and elephantiasis in over 40 million people around the world (Ottesen et al., 2008). It is estimated that $2 \%$ of the world's population is infected by lymphatic filariasis and more than one billion people (20\% of world's population) are at risk of acquiring infection (WHO, 2011). The infection can be treated with drugs. However, chronic conditions may not be curable by anti-fi- larial drugs and require other measures, e.g. surgery for hydrocele, care of the skin and exercise to increase lymphatic drainage in lymphoedema. Annual treatment of all individuals at risk (individuals living in endemic areas) with recommended anti-filarial drugs, diethyl - carbamazine citrate (DEC) and albendazole, or ivermectin and albendazole; or the regular use of DEC fortified salt can prevent occurrence of new infection and disease (Hoerauf, 2008).

Owing to the complex nature of parasites, the immune system is confronted with a highly diverse antigen repertoire, which is perpetuated by a number of biological characteristics of the helminths. Vaccination against parasites is still not a reality, despite 
decades of research. Strategies to identify candidate vaccine antigens against brugian or bancroftian filariasis include screening expression libraries with immune sera (Freedman et al., 1989), differential screening of abundantly expressed mRNAs (Gregory et al., 2000) or by the Expressed Sequence Tag (EST) approach (Blaxter et al., 1999). These strategies have facilitated the identification of several potential vaccine candidates offering varying degrees of protection against filarial infection in animal models (Thirugnanam et al., 2007; Anand et al., 2008; Vanam et al., 2009; Dakshinamoorthy et al., 2013). Antioxidant enzymes thioredoxin (TRX), thioredoxin peroxidase (TPX), glutathione-S-transferase (GST), superoxide dismutase (SOD) and glutathione peroxidase (GPX), most of which are present in all stages of the parasite and have been involved to protect the parasites from the host are reported as promising vaccine candidates (Maizels et al., 2001; Veerapathran et al., 2009; Madhumathi et al., 2010).

A new approach in vaccine technology is using recombinant protein subunits as peptide vaccines. Synthetic peptide vaccines and B-T cell chimera's containing immunodominant regions from antigens have been developed for malaria (Nardin et al., 2001), Schistosomiasis (Arnon et al., 2000) and many other viral and parasitic diseases. Thioredoxin and transglutaminase, though being reported as promising vaccine candidates cannot be used as whole antigen due to the homology that it shares with host proteins (Kunchithapautham et al., 2003; Vanam et al., 2009; Madhumathi et al., 2010). Hence immunologically dominant B and T epitopes from these proteins were identified and constructed as Multi antigen peptide on an inert lysine core.

Adjuvants are usually defined as compounds that can increase and/or modulate the intrinsic immunogenicity of an antigen. Adjuvants are molecules, compounds or macromolecular complexes that boost the potency and longevity of specific immune response to antigens, but cause minimal toxicity or long lasting immune effects on their own (Wack, 2005). Alum is the universally licensed adjuvant so far used in humans approved by the Food and Drug Administration (FDA). Aluminum adjuvants selectively enhance the type 2 immune response. Although alum is able to induce a good antibody $\left(\mathrm{T}_{\mathrm{H}} 2\right)$ response, it has little capacity to stimulate cellular $\left(T_{H} 1\right)$ immune responses which are important for protection against many pathogens. Alum has potential to cause severe local and more serious systemic side- effects. (Petrovsky \& Aguilar, 2004). Hence, there is demand for safe and non-toxic adjuvants which are able to stimulate cellular $\left(T_{H} 1\right)$ immunity.

$\mathrm{N}$-acetylmuramyl-L-alanyl-D-glutamic acid (AcMur-L-Ala-D-Glu), a synthetic analog of Muramyl Di Peptide (MDP) increases the humoral immune response when given in aqueous media instead of the usual water-in-oil emulsion (Audibert et al, 1976). N-acetylmuramyl-L-alanyl-D-isoglutamine (MDP for muramyl dipeptide), a component of many bacterial cell wall peptidoglycans has a strong immunomodulatory activity and have been utilized in preparation of certain synthetic vaccines (Ellouz et al., 1974). MDP as well as its synthetic analogues possess direct mitogenic activity and the ability to polyclonally activate murine spleen cells in vitro under certain conditions (Wood \& Staruch, 1980, Löwy et al, 1980). The present study focusses the immune response of Thioredoxin Transglutaminase Multi Antigen Peptide (TT MAP) which encompasses 3 immunodominant peptides (2 from thioredoxin and one from transglutaminase) formulated with MDP as an adjuvant in mice model. This formulation is also compared with TT MAP immunised with alum as adjuvant.

\section{Materials and Methods}

\section{Synthesis of MAP}

The chimeric MAP is a grouping of heteromeric/homomeric immunodominant peptide sequences. The synthesis is done using standard Fmoc chemistry. Briefly, the Fmoc-Gly-HMP-TentaGel resin, substitution factor $0.16 \mathrm{mmol} / \mathrm{g}$, was pre-swelled in dichloromethane (DCM) for 10 min as per the manufacturer's instructions and kept for swelling in dimethylformamide (DMF) overnight. The F-moc group was removed using $20 \%$ piperidine in DMF. To this, F-mocLys-(ivDde) was attached. The N-terminal F-moc is removed and the peptide was synthesized. At the end of the sequence, the last amino acid used was with a different protecting group, t-Boc, in order to prevent its removal while deprotecting F-moc groups during attachment of different sequences and to prevent unwanted chain elongation thus end capping the synthesized peptide sequences. In the next step, the $\xi-\mathrm{NH}_{2}$ protecting group, ivDde is removed using $2 \%$ hydrazine in DMF and another Fmoc-Lys-(ivDde) was attached. The cycle was repeated till the completion of all the sequences. The epitopic sequences are given in Table 1.

The peptide sequences were synthesized by solid phase technique, using f-moc chemistry and assembled on Tentagel resin (Sigma-Aldrich) as TRX-TGA MAP (TT MAP) constructs. The MAP construct were purified and the purity was analyzed by high performance liquid chromatography.

Table 1. Epitope sequences in Thioredoxin Transglutaminase Multi Antigen Peptide (TT MAP)

\begin{tabular}{ll}
\hline Antigens & Epitope Sequences \\
\hline Transglutaminase peptide & KEFLLHETNGLVGIRTSENRYQFD \\
Thioredoxin peptide 1 & ADLLANINLKKADGTVKKGSDALANK \\
Thioredoxin peptide 2 & SEIEKLKNKYEVAGIP \\
\hline
\end{tabular}

Expression and purification of recombinant WbTransglutaminase (rTGA) and recombinant BmThioredoxin (rTRX)

The partial sequence of $W b$-TGA was cloned in T7 expression vector pRSETB (Invitrogen, Carlsbad, CA), with an N-terminal histidine tag, were expressed in E.coli GJ- 1158. Bacterial cell cultures were induced for 4 hours with $150 \mathrm{mM} \mathrm{NaCl}$ at an O.D of 0.6. The recombinant protein were analysed on $12 \%$ SDS-PAGE and the electro-transferred protein bands on to the nitro cellulose membrane were further analysed by Western blot analysis. Anti-TGA antibody 
raised in mice (1:10000 dilutions) and alkaline phosphatase labelled goat anti-mouse antibody were used as primary and secondary antibodies, respectively, for the immunoblot analysis. Bacterial culture was subjected to cell lysis by sonication. The recombinant protein was present in the insoluble fraction revealing its property to form inclusion body. The insoluble fraction was washed twice with cell lysis buffer (50 mM Tris-Cl, $100 \mathrm{mM} \mathrm{NaCl}, 0.5 \%$ (v/v) Triton $\mathrm{X}-100,1 \mathrm{M}$ urea) $\mathrm{pH} 8.0$, centrifuged at $12000 \mathrm{~g}$ for $15 \mathrm{~min}$. The pellets were then resuspended in $3 \mathrm{M}$ urea in buffer $(50 \mathrm{mM}$ Tris, $200 \mathrm{mM} \mathrm{NaCl}, \mathrm{pH}$ 8.2) overnight, centrifuged at $15000 \mathrm{~g}$ for $15 \mathrm{~min}$. The solubilised protein in supernatant was purified using chelating sepharose fast flow (Amersham Biosciences, Uppasala, Sweden). After washing this column with the same buffer containing urea, the recombinant proteins were eluted with the same buffer containing $150 \mathrm{mM}$ imidazole. The eluted proteins were loaded on to a desalting column (GE Healthcare-Lifesciences) for further purification.

The Bm-TRX-1 gene in pRSET B vector was expressed in $E$. coli (GJ1158) using $100 \mathrm{mM} \mathrm{NaCl}$ at 0.6 O.D. for $3 \mathrm{~h}$. The soluble protein was purified in non-denaturing conditions by ion exchange chromatography using DEAE Sepharose and analyzed on $12 \%$ SDS-PAGE. The protein was further analyzed using mouse anti-TRX sera.

Protein concentrations were estimated by Bradford assay (Biorad, CA, USA) and then used for immunization.

\section{Immunization in Mice}

Six to eight weeks old female BALB/c $\left(\mathrm{H}-2^{\mathrm{d}}\right)$ were procured from TamilNadu Veterinary and Animal Sciences University, Chennai. All experiments were performed in accordance with 'Indian
Animal Ethics Committee' regulations. A dosage of $50 \mu \mathrm{g}$ of TT MAP/100 $\mu$ l PBS was given for each mouse in TT MAP groups. TT protein groups received $12.5 \mu \mathrm{g}$ each of rTRX \& rTGA (total $25 \mu \mathrm{g} / 100 \mu \mathrm{l}$ PBS for each mouse) as cocktail vaccine. Individual proteins TRX and TGA ( $25 \mu \mathrm{g} / 100 \mu$ l PBS for each mouse) were immunised with MDP/Alum as separate groups which served as whole protein controls. Aluminium hydroxide gel \& MDP (Sigma Aldrich, USA)) were admixed to each dose. $5 \mu \mathrm{g}$ of MDP/mice was given for each dose. In case of alum, a 1:1 ratio was given. Four doses at weekly intervals were administered intra muscularly for MDP groups and intra peritoneally for alum groups. Intramuscular immunizations were carried out by injection of $100 \mu$ volumes in the hind right leg ventral muscle. The adjuvant control group received Phosphate Buffered Saline alone in MDP/alum. Sera collected periodically after immunization in mice was used to check the antibody titer by ELISA. Immunizations of all the groups were performed during the same period. Booster doses were given on days 14,21 and 28 .

\section{Determination of Antibody titer}

The sera were collected periodically after immunization and stored at $-80{ }^{\circ} \mathrm{C}$ and the antibody titer was determined by ELISA, in which 96-well microtiter plates (Immulon2, Dynatech,VA, USA) were coated with $100 \mathrm{ng} /$ well of respective antigen. The plates were blocked with $5 \%$ skimmed milk, after washing with PBST and PBS, a 2 fold serial dilution from 1:500 to 1:64000 were performed using respective antisera. The antibody titer was determined by fixing a cut-off value which was obtained by the mean plus $3 S D$ of the OD value of pre-immune serum. The highest dilution of the antiserum that 1a)

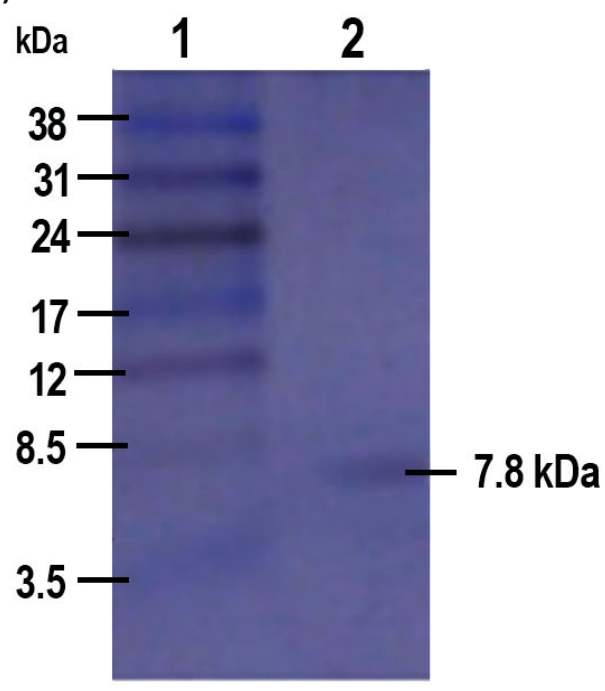

1b)

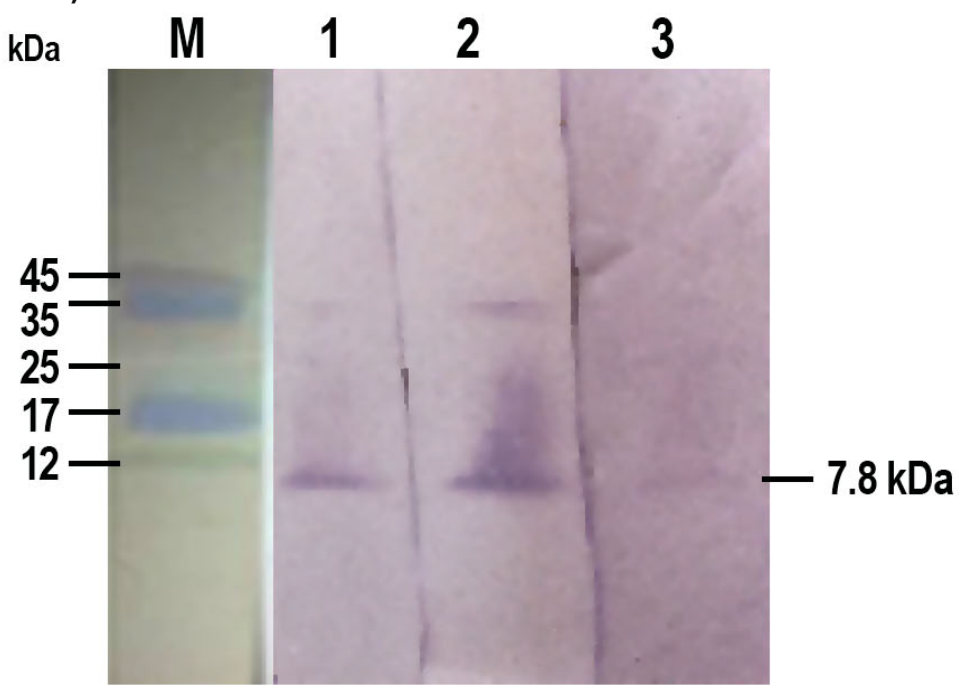

Fig. 1. Thioredoxin-Transglutaminase Multi Antigen Peptide (TT MAP):

1a. SDS-PAGE analysis of the synthetic TT MAP antigen: Lane 1 - Molecular marker, Lane 2 -TT MAP.

1b. Immunoblot analysis of TT MAP peptide: Lane M - Molecular marker, Lane 1-3 - Cross-reactivity of TT MAP with antibodies raised in mice against individual peptides (Bm TGA P1, Bm TRX P1 and Bm TRX P2, respectively) 
2a)

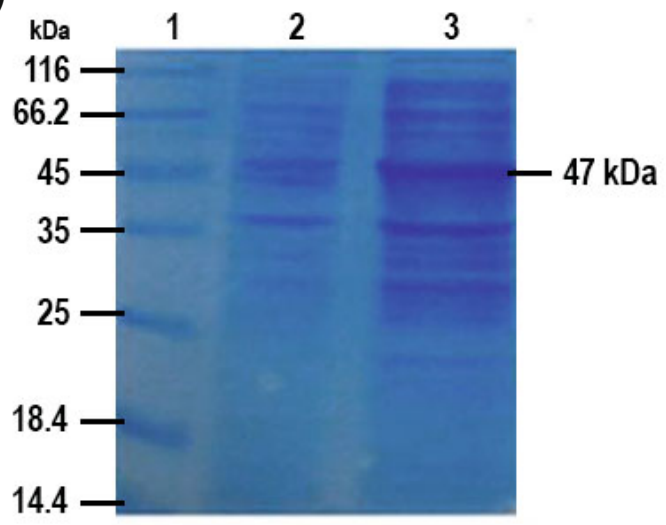

2b)

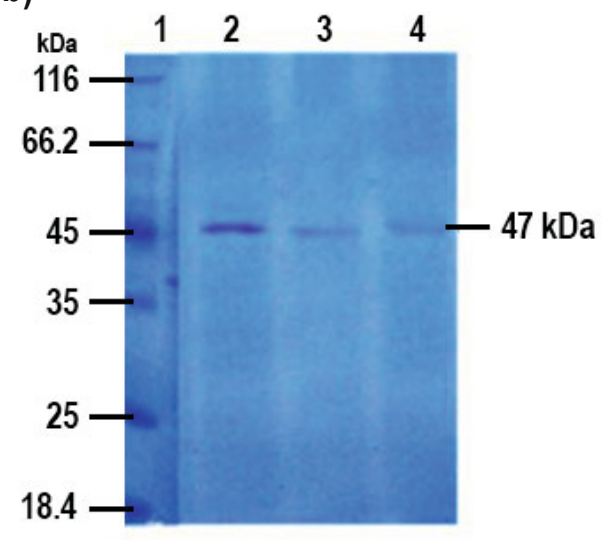

2c)

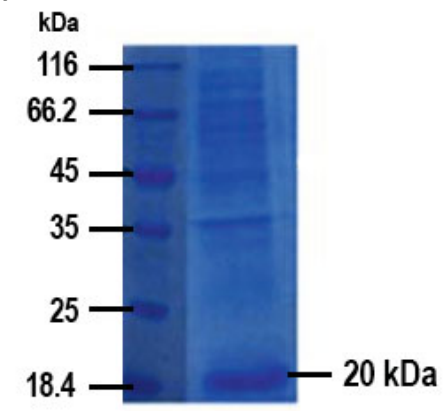

2d) 2e)

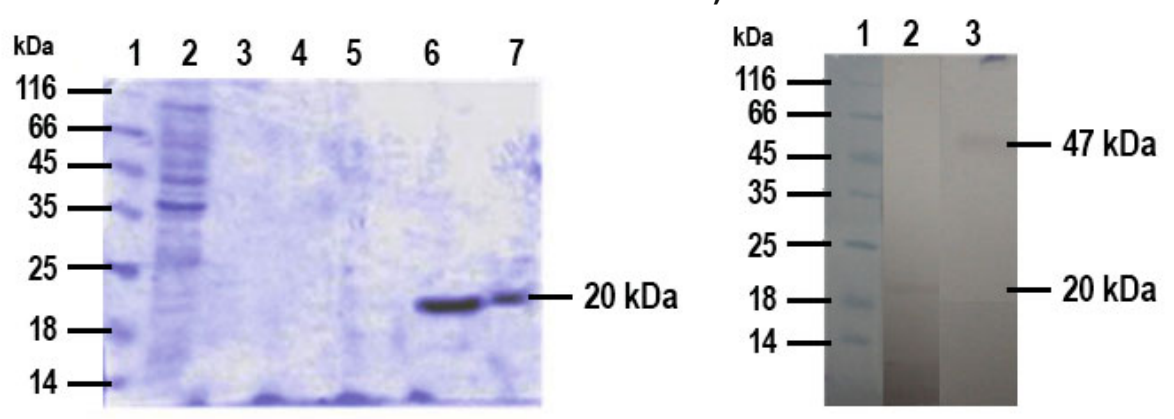

Fig. 2. Expression and purification of recombinant proteins:

(2a) Expression of the rTGA analyzed by $12 \%$ SDS gel: Lane 1- Molecular marker, Lane 2 - pRSETB TGA uninduced, Lane 3 - pRSETB TGA induced (2b) Purification of rTGA analyzed by $12 \%$ SDS gel: Lane 1 - Molecular marker, Lanes 2-4 Pure TGA elutes

(2c) Expression of rTRX analyzed by $12 \%$ SDS gel. Lane 1 - Molecular marker, Lane 2 - Expression of the $20 \mathrm{kDa}$ rTRX produced from E. coli (GJ1158) (2d) Purification of rTRX analyzed by $12 \%$ SDS gel: Lane 1- Molecular marker, Lane 2- Flow through elute of TRX, Lanes 3-7 - Pure TRX elutes

(2e) Immunoblot cross-reactivity of purified rTRX and TGA with anti filarial mouse serum: Lane 1 - Molecular marker, Lane 2 - immunoblot with anti-TRX sera, Lane 3 - immunoblot with anti-TGA sera

showed an OD value above the cut-off value was taken as the antibody titer. The colour developed using mouse antibody conjugated with alkaline phosphatase/p-nitrophenyl phosphate as substrate $(1 \mathrm{mg} / \mathrm{ml})$ in substrate buffer (100 mM Tris-Cl, pH 9.5, $100 \mathrm{mM}$ $\mathrm{Nacl}, 5 \mathrm{mM} \mathrm{MgCl}_{2}$ ) and the absorbance was read at $405 \mathrm{~nm}$.

For determination of antibody isotypes, the sera (dilution1:500) from different immunization groups of mice were incubated for $1 \mathrm{~h}$ at $37^{\circ} \mathrm{C}$, with respective proteins ( $100 \mathrm{ng}$ of TRX/TGA for whole protein groups and $50 \mathrm{ng}$ of TRX and $50 \mathrm{ng}$ of TGA for TT protein groups and $100 \mathrm{ng}$ of TT MAP for MAP immunised groups) coated on ELISA plates. The IgG isotype binding was detected using secondary rabbit anti-mouse lgG antibody specific for each subclass (Sigma, USA) as per the manufacturer instructions. The absorbance was read at $405 \mathrm{~nm}$.

\section{Splenocyte Proliferation}

Immunized mice were splenectomised aseptically and the splenocytes were separated and washed twice with fresh culture medium
(RPMI 1640 from Gibco BRL, USA). Lysis buffer (0.1\% ammonium chloride) was added to the pellet to remove the red blood cells after which the lymphocytes were counted. The single cell suspension was cultured in triplicate in 96 well plates at $0.2 \times 10^{6}$ cells/ml in RPMI 1640 medium (100 $\mu \mathrm{l} /$ well) supplemented with gentamycin ( $80 \mu \mathrm{g} / \mathrm{ml}), 25 \mathrm{mM}$ HEPES, 2 mM glutamine and $10 \%$ fetal bovine serum. The cells were then stimulated in vitro with respective antigen, a positive control Concanavalin (Con A) $(1 \mu \mathrm{g} /$ well each). Wells with medium alone were used as unstimulated controls. After growth at $37{ }^{\circ} \mathrm{C}$ in a $\mathrm{CO}_{2}$ incubator for $72 \mathrm{~h}$, the cultures were pulsed with $\left(\mathrm{H}_{3}\right)$ thymidine (USB, Amersham Pharmacia, UK) at $0.5 \mu \mathrm{Ci} /$ well. The cells were incubated for $18 \mathrm{~h}$ and harvested onto glass fiber disks and thymidine incorporation was measured by a liquid scintillation counter. All cultures were set up in triplicate and the results were expressed as mean stimulation index $(\mathrm{SI})=($ counts per minute of stimulated cultures/ counts per minute of unstimulated cultures) $\pm S D$. 


\section{Analysis of cytokines}

Cytokine levels of the various immunised groups of mice were analysed. $5 \times 10^{6}$ cells (lymphocytes obtained from immunised mice)/ $\mathrm{ml}$ were stimulated with respective antigens. The culture supernatants were centrifuged at $5000 \mathrm{rpm}$ for $15 \mathrm{~min}$, filtered through $0.22-\mu \mathrm{m}$ pores and assayed for cytokine levels using sandwich ELISA for anti-mouse IL-2, IL-4, IL-5, IL-10 and IFN- $\gamma$ (e-Biosciences) according to the manufacturer's instructions. All concentrations were derived from standard curves and data expressed in picograms $/ \mathrm{ml}$.

\section{Statistical Analysis}

The statistical analysis of the data by One-way or Two-way ANOVA with Bonferroni post-test were performed using GraphPad Prism software (version 5.03). Probability $(p)$ value of $<0.05$ was considered statistically significant.

\section{Results}

\section{Synthesis of TT MAP}

The Thioredoxin-Transglutaminase Multi Antigen Peptide (TT MAP) used in this study comprises of a tri-peptide, one epitope from Brugia malayi Transglutaminase (TGA) (amino acids 203-226 named as TGA P1) and two epitopic portions from Brugia malayi Thioredoxin (TRX) (amino acids 2-27 named as TRXP1 and amino acids 94-107 named as TRX P2) attached to an inert lysine core. The three peptides were chosen to be synthesised as MAP based on previous studies in mice models (Madhumathi et al., 2010) with individual peptides (Data not shown). All the 3 peptides are bonded to the inert lysine core. The chemically synthesised TT MAP has a molecular weight of $7.8 \mathrm{kDa}$ which is shown in Tricine SDSPAGE (Fig. 1a) and confirmed by immunoblot (Fig. 1b).

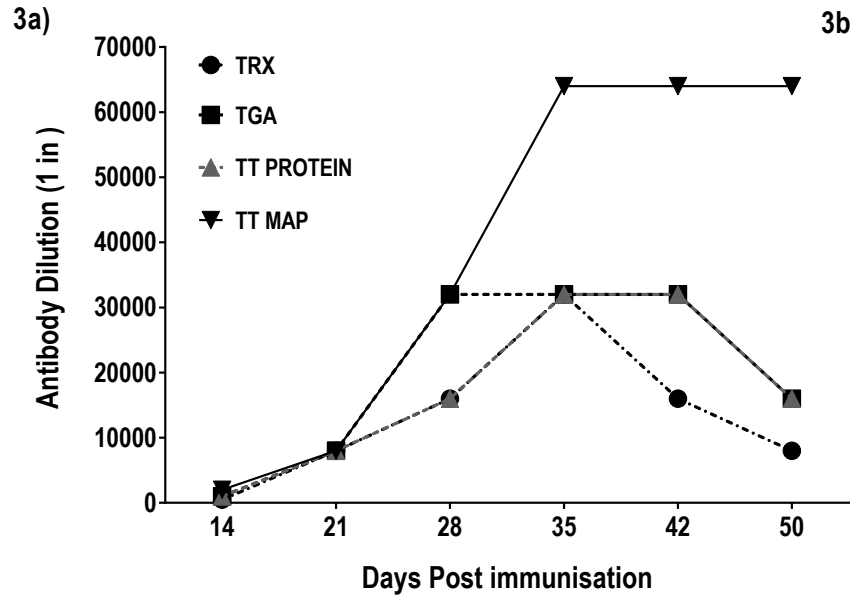

b)

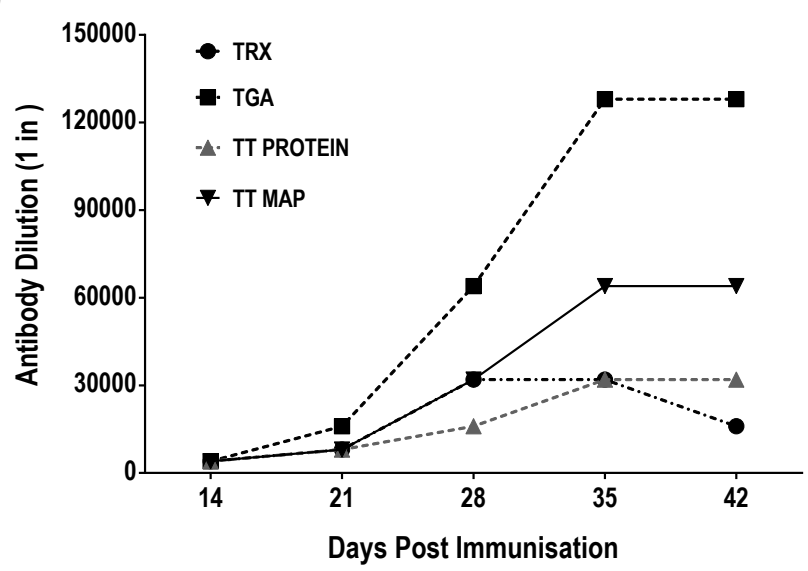

3c)

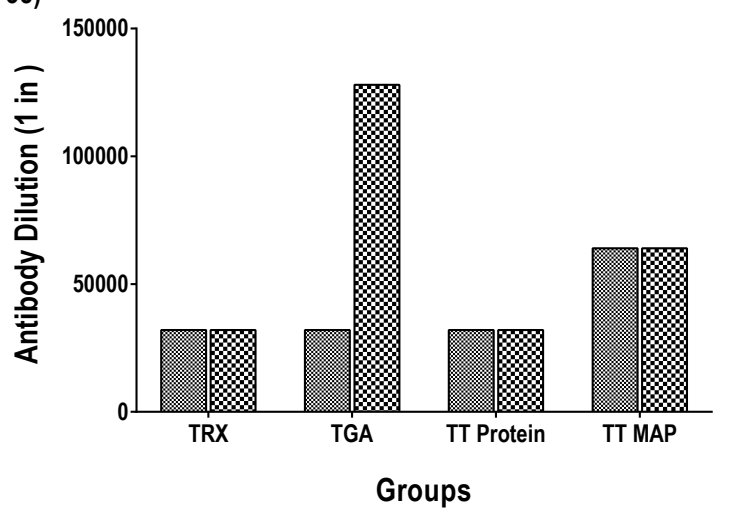

Alum

冈 MDP

Fig. 3. Humoral immune response of TT MAP with Alum and MDP

3a. Antigen specific antibody titers (in thousands) in mice immunised with alum as adjuvant at day 14, 21, 35 and 42 post-immunization.

3b. Antigen specific antibody titers (in thousands) in mice immunised with MDP as adjuvant at day 14, 21, 35 and 42 post-immunization.

3c. Comparison of peak antibody titers followed by immunization with TRX, TGA, TT protein and TT MAP with alum/MDP as adjuvants. Individual points represent mean peak titers determined on day 35 ( $n=5$ mice per experimental group) 
4a)

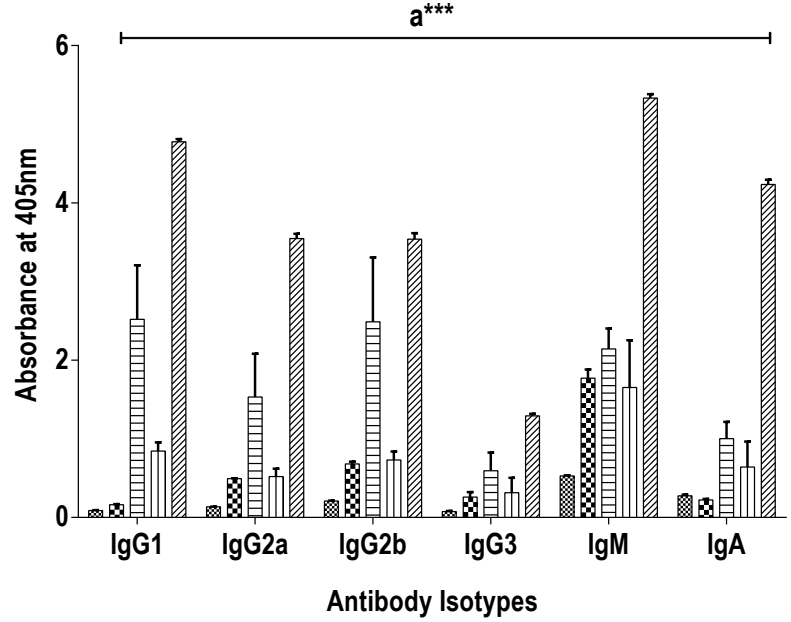
Alum control
8 TRX
目 TGA
미 TT Protein
TT MAP

4b)

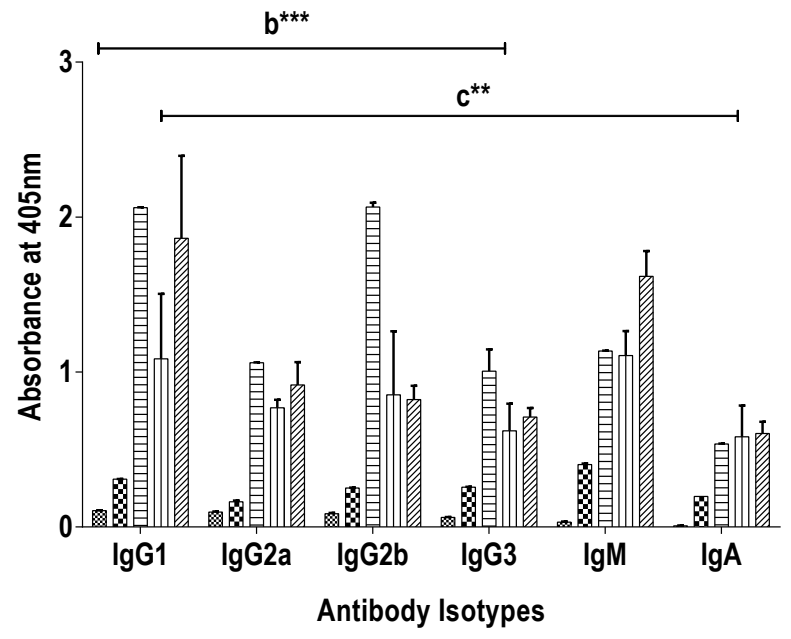

MDP Control $\otimes$ TRX $\quad$ 目 TGA

Fig. 4. Immunglobulin isotype profiles detected after the immunizations with Alum or MDP adjuvant

4a. Immunoglobulin isotype profile (IgG1, IgG2a, IgG2b, IgG3, IgM, IgA) followed by immunization with TRX, TGA, TT protein and TT MAP with alum as adjuvant. 4b. Immunoglobulin isotype profile (IgG1, IgG2a, IgG2b, IgG3, IgM, IgA) followed by immunization with TRX, TGA, TT protein, TT MAP with MDP as adjuvant. The antibody isotypes measured in 1:500 diluted sera are shown in bars. Data represents mean titer $\pm S D$ ( $n=5$ mice per experimental group). Asterisks represents level of significance between control (adjuvant) and immunised groups ( ${ }^{* \star *} P<0.001$; ** $P<0.01$ )

Expression and Purification of recombinant proteins TRX and TGA The rTGA was expressed as $47 \mathrm{kDa}$ protein and rTRX was expressed as a $20 \mathrm{kDa}$ protein without histidine tag (Fig. 2a, 2c). Both the proteins were expressed in E. coli (GJ1158) Recombinant TGA was obtained in the insoluble fraction and hence the lysate was subjected to urea solubilisation which was then purified by Immobilised Metal Affinity Chromatography (Fig. 2b). Recombinant TRX was purified in the non- denatured form by Anion exchange chromatography (Fig. 2d). The purified proteins were then confirmed by mouse anti TGA and anti TRX sera (Fig. 2e).
Measurement of antibody by ELISA

Sera from mice were collected after each immunization and were used to measure the total lgG responses by ELISA. The antibody response was measured in terms of peak titers. Although the peak titers for TT MAP with MDP and alum were the same (1 in 64000) MDP group immunised with Individual proteins (TRX/TGA) showed a higher titer when compared with alum. TGA alone elicited a higher titre with MDP (1 in 1, 28,000) whereas TGA+alum elicited a peak titre of 1 in 32,000. MDP in combination with TRX elicited a peak titre of 1 in 32,000 which eventually dropped to 


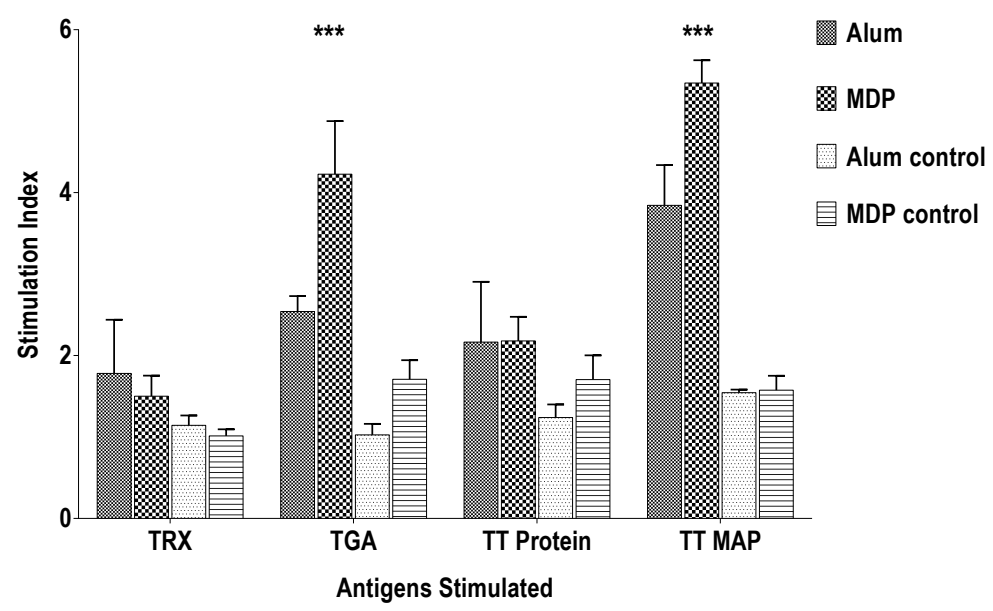

Fig. 5. Splenocyte proliferation

Splenocyte proliferation in different mice groups stimulated with corresponding antigens TRX, TGA, TT protein, TT MAP, compared to MDP/Alum controls. Data are presented as mean $( \pm$ SD) Stimulation Index (S.I.) of five mice per experimental group. Asterisks represents level of significance between the TGA and TT MAP group where the MDP was used as adjuvant $\left({ }^{* *} \mathrm{P}<0.001\right)$

16,000 on $42^{\text {nd }}$ day (Fig. 3a, 3b). The isotype profile of TT MAP with alum/MDP showed a similar pattern with a higher lgG1 response. TT MAP with alum shows a significant increase of all isotypes as compared to the individual and TT Protein groups immunised with alum ( $a^{* * *}, P<0.001$ in Fig. 4a). In case of MDP as adjuvant, TGA immunised group shows a significant increase in IgG2b levels as compared to other groups ( $b^{* * *}$ in Fig $4 b$ ). The lgG1 levels of TGAMDP was in par with TT MAP immunised with MDP. The isotype levels of TT MAP was significant $\left(C^{* *}, P<0.01\right)$ as compared to TT protein group immunised with MDP.

\section{Cellular Immune response}

The cell-mediated lymphoproliferative responses when examined using the splenocytes from each animal in proliferation assays, the maximum proliferation was observed for TT MAP with MDP. (Fig. 5). The difference in splenocyte proliferation was well pronounced between alum and MDP immunised groups $(P<0.001)$. The levels of the cytokines IL-2, IL-4, IL-5, IL-10, and IFN- $\gamma$ were measured, to understand the cytokines involved in the cell-mediated pathway (Table 2).The IL-2 and IFN- $\gamma$ levels in TT MAP with MDP immunized groups is higher than all the alum immunized groups. IL-4 levels in TT MAP with alum immunised groups were significantly higher than TT MAP with MDP group whereas there existed no significance in IL-5 levels of TT MAP groups. IL-10 level in TT MAP with MDP is significantly lower in comparison with alum immunised groups as wells as protein immunised groups with MDP/alum.

\section{Discussion}

Filariasis is considered one of the most complex infections of humans, and sufficient immunoprophylactic studies for lymphatic filariasis has not been carried out. The widespread chronic infection and the morbidity are due to the spectrum of immune evasion strategy developed by the filarial nematode. Mouse models provide us with the insight into the possible mechanism by which the filarial nematode can be destroyed (Lawrence \& Devaney, 2001).

\begin{tabular}{lcccccc}
\multicolumn{7}{c}{ Table 2. Cytokine levels } \\
\hline Groups & \multicolumn{1}{c}{ IL2 } & IFN-G & IL-4 & IL-5 & IL-10 \\
\hline \multirow{2}{*}{ TRX } & Alum & $98 \pm 10.6$ & $160.81 \pm 12.21^{\mathrm{a}}$ & $321 \pm 3.11^{\mathrm{a}}$ & $230.33 \pm 6.55^{\mathrm{a}}$ & $89.27 \pm 2.29$ \\
& MDP & $144.25 \pm 1.76^{\mathrm{a}}$ & $100.81 \pm 3.21$ & $259.29 \pm 0.41$ & $116.53 \pm 3.68$ & $110.47 \pm 1.45^{\mathrm{a}}$ \\
TGA & Alum & $177.35 \pm .88$ & $375.81 \pm 6.423$ & $509 \pm 2.49$ & $216.85 \pm 6.766$ & $61.11 \pm .66$ \\
TT & MDP & $199.25 \pm 5.3^{\mathrm{a}}$ & $367.45 \pm 3.21$ & $547.38 \pm 1.35$ & $336.30 \pm 3.9^{\mathrm{a}}$ & $90.64 \pm 0.06^{\mathrm{a}}$ \\
Protein & Alum & $127.37 \pm 0.88$ & $230.54 \pm 1.28^{\mathrm{a}}$ & $329.88 \pm 6.66^{\mathrm{a}}$ & $283.88 \pm 0.922$ & $94.44 \pm .54$ \\
TT MAP & MDP & $159.25 \pm 1.78^{\mathrm{a}}$ & $150.81 \pm 5.78$ & $293.11 \pm 4.57^{\mathrm{b}}$ & $263.95 \pm 6.55$ & $132.62 \pm 7.25^{\mathrm{a}, \mathrm{b}}$ \\
& Mlum & $149.25 \pm 8.83$ & $214 \pm 2.86$ & $341 \pm 3.26^{\mathrm{a}}$ & $293 \pm 5.48$ & $85 \pm 4.32^{\mathrm{a}}$ \\
\hline
\end{tabular}

Cytokine levels $(\mathrm{pg} / \mathrm{ml})$ in culture supernatants of spleen cells, from mice, immunized with the TRX, TGA, TT Protein or TT MAP with MDP as adjuvant and stimulated in vitro with respective antigens. Experiments were done thrice and data represented as mean concentration $\pm S D$.

$(a=P<0.005$, Comparison of individual groups of alum and MDP; $b=P<0.005$, Comparison of TT Protein MDP and TT MAP MDP groups) 
Metabolically active enzymes are highly conserved and hence they share homologous regions with host proteins which may show cross reactivity when developed as vaccines (Madhumathi et al., 2011). The sequence similarity of filarial TRX and TGA with host proteins is a major drawback towards the exploitation of their potent immunogenicity to be developed as vaccines. Apart from the homologous sequence, the immuno-modulatory effect of TRX reported by Kunchithapautham et al. (2003) sets another limitation for TRX to be used as a vaccine candidate. Hence, epitopic portions which were non homologous to the host proteins (Madhumathi et al., 2010) and were highly reactive to the sera of putatively immune individuals (Endemic normal) were chosen to be constructed as Multi Antigen Peptides on an inert lysine core.

The present study which focuses on the immune responses of TT MAP formulated with MDP is an attempt to improve the vaccine efficacy of TT MAP. MDP, the synthetic analogue of the bacterial peptidoglycan moiety has been reported as safe and potent adjuvant in various immunisation studies (Parant, 1979; Moschos et al., 2005). TT MAP-MDP showed a higher antibody response in comparison with TRX and TT protein cocktail mixture. IL-10 levels in TRX immunised mice seem to be higher, predicting the regulatory $T$ cell effect which may have down regulated the humoral immune response in TRX immunised groups. MDP is Nucleotide Oligomerization Domain containing protein (NOD) dependent and activates the NF-KB for the stimulation of immune responses. Filarial nematode-derived thioredoxin influences the host immune cell activity by inhibiting the activity of p38 MAP kinase (Hashimoto et al., 1999). The immuno-modulatory effect of TRX well correlates with the mechanisms predicted by Kunchithapautham et al. (2003). On the contrary TT MAP that constitutes 2 immunodominant epitope from TRX has elicited a higher antibody response which shows that the epitopic portions are devoid of immuno-modulating sequences which may have inhibited the adjuvant potency. The antibody isotype elicited by TT MAP with alum/MDP showed similar patterns of isotype profile. IgG1 and lgM levels were higher in TT MAP immunized groups. The high levels of IgM antibodies indicate host defense mechanism. IgM and IgG1 have been implicated in protection in experimental filariasis (Thirugnanam et al., 2007; Rajan et al., 2005). T cell responses for both MDP and Alum with TT MAP seemed to have elicited different $T$ helper response which is due to the immuno modulating activity contributed by the adjuvants. TT MAP formulated with alum had a biased $\mathrm{T}_{\mathrm{H}} 2$ response whereas TT MAP formulated with MDP shows a balanced $T_{H} 1 / T_{H} 2$ response. Previous experiments on MDP has reported on the elicitation of cytokines in vitro mediated by chemical analogues of MDP (Gobec et al., 2004), where some resulted in type I cytokine secretion but others resulted in more balanced cytokine profiles (Moschos et al., 2005). Vaccines formulated with MDP when combined with liposomes may enhance higher cell mediated immune responses. Studies have confirmed that alum selectively promotes $\mathrm{T}_{\mathrm{H}} 2$ responses in mice, including $\mathrm{IL}-4$ production and generation of IgG1 antibodies (Brewer et al., 1999) which also evident in the present study. Alum usually generates $\mathrm{T}_{\mathrm{H}} 2$-related specific immune responses. In part this bias is caused by alum's ability to induce production of IL-4. IL-4 made in response to alum is not required to drive $T_{H} 2$ responses but rather, acts by suppressing $T_{H} 1$-related phenomena, leading to a more polarized immune response (McKee et al, 2009). The experiments described here demonstrated that TT MAP administered with MDP elicited TT MAP specific $T_{H} 1$ and $T_{H} 2$ cells, since both $T_{H} 1$ (IFN- $\gamma$ )-and $T_{H} 2$ (IL-4 \& IL-5) type cytokines were secreted in response.

In conclusion, our findings with MDP as adjuvant administered with TT MAP via intramuscular route in Balb/c mice model was safe and the formulation elicited a higher antibody response with a balanced $T_{H} 1 / T_{H} 2$ response. However the assessment of this formulation capable of contributing to protective immune response could be confirmed only after immunisation and challenge studies carried out in permissive animal models.

\section{Acknowledgements}

The authors are thankful to Department of Biotechnology (DBT), Govt. of India for their financial assistance. The Senior Reseach Fellowship to Ms.Christiana from CSIR is gratefully acknowledged.

\section{References}

Anand, S.B., Murugan, V., Prabhu, P.R., Anandharaman, V., Reddy, M.V., KalIRAJ, P. (2008): Comparison of immunogenicity, protective efficacy of single and cocktail DNA vaccine of Brugia malayi abundant larval transcript (ALT-2) and thioredoxin peroxidase (TPX) in mice. Acta Trop., (107)2: 106 - 112. DOI: 10.1016/j.actatropica.2008.04.018

Arnon, R., Tarrab-Hazdal, R., Steward M. (2000): A mimotope peptide-based vaccine against Schistosoma mansoni: synthesis and characterization. Immunology, (101): 555 - 562. DOI: 10.1046/j.1365-2567.2000.00139.x

Audibert, F., Chédid, L., Lefrancier, P., Choay. J. (1976): Distinctive adjuvanticity of synthetic analogs of mycobacterial water-soluble components. Cell. Immunol., 21(2) $243-249$

Blaxter, M., Aslett, M., Guiliano, D., Daub, J. (1999): Parasitic helminth genomics. Filarial Genome Project. Parasitology, 118: S39 - S51

Brewer, J.M., Conacher, M., Mohrs, M., Brombacher, F., AlexanDER, J. (1999): Aluminium hydroxide adjuvant initiates strong antigen-specific Th2 responses in the absence of IL-4- or IL-13-mediated signalling. J. Immunol., 163: $6448-6454$

Dakshinamoorthy, G., Samykutty, A.K., Munirathinam, G., Reddy, M.V., Kalyanasundaram, R. (2013): Multivalent fusion protein vaccine for lymphatic filariasis. Vaccine, 31(12): 1616 - 1622. DOI: 10.1016/j.vaccine.2012.09.055

Ellouz, F., Adam, A., Ciorbaru, R., Lederer, E. (1974): Minimal structural requirements for adjuvant activity of bacterial peptidoglycan derivatives. Biochem. Biophys. Res. Commun., 59(4): 1317 - 1325. DOI: 10.1016/0006-291X(74)90458-6 
Freedman, D.O., Nutman, T.B., Ottesen, E.A. (1989): Protective immunity in Bancroftian filariasis- Selective recognition of a $43-\mathrm{kDa}$ larval stage antigen by infection free individuals in an endemic area. J.Clin. Invest., (83): $14-22$

Gobec, S., Sollner-Dolenc, M., Urleb, U., Wraber, B., Simcic, S., FILIPIC, M. (2004): Modulation of cytokine production by some phthalimido-desmuramyl dipeptides and their cytotoxicity. Farmaco, 59(5): 345 - 352. DOI: 10.1016/j.farmac.2004.01.003

Gregory, W.F., Atmadja, A.K., Allen, J.E., Maizels, R.M. (2000): The abundant larval transcript $1 / 2$ genes of Brugia malayi encode stage-specific candidate vaccine antigens for filariasis. Infect. Immun., (68): 4174 - 4179. DOI: 10.1128/IAI.68.7.4174-4179.2000 Hashimoto, S., Matsumoto, K., Gon, Y., Furuichi, S., Maruoka, S., TAKeshitA, I., HIROTA, K., Yodol, J., Horie, T. (1999): Thioredoxin negatively regulates p38 MAP kinase activation and IL-6 production by tumor necrosis factor-alpha. Biochem. Biophys. Res. Commun., 258: 443 - 447. DOI: 10.1006/bbrc.1999.0658

Hoerauf, A. (2008): Filariasis: new drugs and new opportunities for lymphatic filariasis and Onchocerciasis. Curr. Opin. Infect. Dis., 21: 673 - 681. DOI: 10.1097/QCO.0b013e328315cde7

Kunchithapautham, K., Padmavathl, B., Narayanan, R.B., Kaliraj, P., Scott, A.L. (2003): Thioredoxin from Brugia malayi: Defining a 16-Kilodalton Class of Thioredoxins from Nematodes. Infect. Immun., 71: 4119 - 4126. DOI: 10.1128/IAI.71.7.4119-4126.2003 LaWrence, R.A., DeVaney, E. (2001): Lymphatic filariasis: parallels between the immunology of infection in humans and mice. Parasite Immunol., 23: 353 - 361. DOI: 10.1046/j.1365-3024.2001.00396.x LöWy, I., LECLERC, C., CHEDID, L. (1980): Induction of antibodies directed against self and altered-self determinants by a synthetic adjuvant, muramyl dipeptide and some of its derivatives. Immunology, 39 (3): 441 - 450

Madhumathi, J., Anugraha, G., Prince, P.R., Pradiba, D., Kaliraj, P. (2011): Proliferative responses of Brugia malayi TPX-1 and its epitopic peptide ${ }_{29-43}$ in an endemic population of human lymphatic filariasis. Microbes Infect., 13: 602 - 607. DOI: 10.1016/j. micinf.2011.01.008

Madhumathi, J., Prince, P.R., Anugraha, G., Kiran, P., Rao, D.N., Reddy, M.V., KaliRAJ, P. (2010): Identification and characterization of nematode specific protective epitopes of Brugia malayi TRX towards development of synthetic vaccine construct for lymphatic filariasis. Vaccine, 28: 5038 - 5048. DOI: 10.1016/j.vaccine.2010.05.012

Maizels, R.M., Blaxter, M.L., Scott, A.L. (2001): Immunological genomics of Brugia malayi: filarial genes implicated in immune evasion and protective immunity. Parasite Immunol., 23: 327 344. DOI: 10.1046/j.1365-3024.2001.00397.x

McKee, A.S., Munks, M.W., Macleod, M.K.L., Fleenor, C.J., Van Rooljen, N., Kappler, J.W., Marrack, P. (2009): Alum induces innate immune responses through macrophage and mast cell sensors, but these are not required for alum to act as an adjuvant for specific immunity. J Immunol., 183(7): 4403 - 4414. DOI: 10.4049/ jimmunol.0900164

Moschos, S.A., Bramwell, V.W., Somavarapu, S., Alpar, H.O. (2005): Comparative immunomodulatory properties of a chitosan-MDP adjuvant combination following intranasal or intramuscular immunisation. Vaccine, 23(16): 1923 - 1930. DOI: 10.1016/j. vaccine.2004.10.016

Nardin, E.H., Calvo-Calle, J.M., Oliveira, G.A., Nussenzweig, R.S., Schneider, M., Tiercy, J.M., Loutan, L., Hochstrasser, D., Rose, K. (2001): A Totally Synthetic Polyoxime Malaria Vaccine Containing Plasmodium falciparum B cell and Universal T Cell Epitopes Elicits Immune Responses in Volunteers of Diverse HLA types. J. Immunol., 166: 481 - 489. DOI: 10.4049/jimmunol.166.1.481

Ottesen, E.A., Hooper, P.J., Bradley, M., Biswas, G. (2008): The Global Programme to Eliminate Lymphatic Filariasis: Health impact after 8 years. PLoS Negl. Trop. Dis., 2(10): e317. DOI: 10.1371/journal.pntd.0000317

Parant, M. (1979): Biologic Properties of a New Synthetic Adjuvant, Muramyl Dipeptide (MDP). Springer Semin. Immunopathol., 2: 101 - 118. DOI: 10.1007/BF00201976

Petrovsky, N., Agullar, J.C. (2004): Vaccine adjuvants: current state and future trends. Immunol. Cell. Biol., 82(5): 488 - 496. DOI:10.1111/j.0818-9641.2004.01272.x

Rajan, B., Ramalingam, T., Rajan, T.V. (2005): Critical Role for lgM in Host Protection in Experimental Filarial Infection. J. Immunol., 175: 1827 - 1833. DOI: 10.4049/ jimmunol.175.3.1827

Thirugnanam, S., Pandiaraja, P., Ramaswamy, K., Murugan, V., Gnanasekar, M., Nandakumar, K., Reddy, M.V., Kaliraj, P. (2007): Brugia malayi: comparison of protective immune responses induced by Bm-alt-2 DNA, recombinant Bm-ALT-2 protein and prime-boost vaccine regimens in a jird model. Exp. Parasitol., 116: 483 - 491. DOI: 10.1016/j.exppara.2007.02.017

Vanam, U., Pandey, V., Prabhu, P.R., Dakshinamurthy, G., Reddy, M.V., KaliRAJ, P. (2009): Evaluation of immunoprophylactic efficacy of Brugia malayi transglutaminase (BmTGA) in single and multiple antigen vaccination with BmALT-2 and BmTPX for human lymphatic filariasis. Am. J. Trop. Med. Hyg., 80(2): $319-324$

Veerapathran, A., Dakshinamoorthy, G., Gnanasekar, M., Reddy, M.V., Kalyanasundaram, R. (2009): Evaluation of Wuchereria bancrofti GST as a vaccine candidate for lymphatic filariasis. PLoS Negl. Trop. Dis., 3(6): 457. DOI:10.1371/journal.pntd.0000457 Wack, A., RappuOLI, R. (2005): Vaccinology at the beginning of the 21st century. Curr. Opin. Immunol., 17(4): 411 - 418. DOI: 10.1016/j.coi.2005.05.005

WHO (2011): Integrated vector management to control malaria and lymphatic filariasis - WHO Position Statement. Wkly. Epidemiol. Rec., 86(13): 121 - 127

WOOD, D.D., STARUCH, M.J. (1980): Control of the mitogenicity of muramyl dipeptide. Int. J. Immunopharmacol., 3(1): 31 - 44. DOI: 10.1016/0192-0561(81)90043-6 